\title{
Confidence intervals based on resampling methods using Ridge estimator in linear regression model
}

\author{
Yogendra P. Chaubey ${ }^{1}$, Mansi Khurana ${ }^{2}$ and Shalini Chandra ${ }^{3}$ \\ ${ }^{1}$ Concordia University, Montrèal, Canada \\ 2 ICFAI Business School, Gurgaon, India \\ ${ }^{3}$ Banasthali University, Rajasthan, India
}

Received: 8 January 2018, Accepted: 3 April 2018

Published online: 13 December 2018.

\begin{abstract}
In multiple regression analysis, the use of ridge regression estimator over the conventional ordinary least squares estimator was suggested by Hoerl and Kennard in 1970 to beat the problem of multicollinearity that may exist among the independent variables. Keeping this in mind, in the present study, the authors intend to develop and compare different confidence intervals for regression coefficients based on ridge regression estimator using bootstrap and jackknife methodology. For comparison, the coverage probabilities and confidence widths are calculated through a simulation study for the data which suffers from the problem of multicollinearity.
\end{abstract}

Keywords: Regression analysis, multicollinearity, confidence intervals, jackknife technique, bootstrap technique.

\section{Introduction}

In regression analysis, ridge regression estimator (RRE) can be of great use for the estimation of unknown regression coefficients in presence of multicollinearity among the regressors (Hoerl and Kennard [1]). But apart from its ability to create good parameter estimates with smaller mean squared error (MSE) than the usual oridnary least squares estimator (OLSE), it must also provide fine solutions when dealing with more intricate inference problems like obtaining confidence intervals. As the distribution of RRE is completely known when the errors are Gaussian, the use of RRE in finding the confidence intervals for the regression parameters is marred by the fact it may be associated with serious bias and its distribution, specially for non normal errors, may not be easily characterized. Recently, Firinguetti and Bobadilla [2] developed asymptotic confidence intervals for the regression coefficients based on RRE and Edgeworth expansion. Crivelli et. al. [3] proposed the use of a technique that combines the bootstrap and the Edgeworth expansion to obtain an approximation to the distribution of some ridge regression estimators and carried out some simulation experiments.

The most commonly used confidence intervals are approximate confidence intervals which are also known as standard intervals (or normal theory intervals) having the following general form

$$
\hat{\theta} \pm z^{(\alpha)} \hat{\sigma}
$$

where $\hat{\theta}$ is an estimate of the unknown population parameter $\theta$, $\hat{\sigma}$ is the standard error of $\hat{\theta}$, and $z^{(\alpha)}$ is the $100 \alpha^{t h}$ percentile of a normal variate, (for example $z^{(0.95)}=1.645$ etc.). The main drawback of standard intervals is that they are based on an asymptotic approximation that may not be accurate in practice. There has been considerable progress 
on developing better confidence intervals techniques for improving the standard interval, involving bias corrections and parameter transformations. These methods produce approximate confidence intervals that have better coverage accuracy than the standard one. Some references under this area include Efron [4], Hall [5], DiCiccio and Tibshirani [6]. The confidence intervals based on the resampling methods like bootstrap and jackknife can be seen as automatic algorithms for carrying out these improvements. The bootstrap and jackknife are known as the two powerful resampling methods for variance estimation for complex statistics even for non normal errors which is why they could be used for producing confidence intervals. Next section consists of the model, the estimators and the different forms of confidence intervals based on bootstrap and jackknife methods.

\section{The model, estimators \& confidence intervals}

Consider the following multiple linear regression model

$$
y=X \beta+u
$$

where $y$ is an $n \times 1$ vector of observations on the variable to be explained, $X$ is an $n \times p$ matrix of $n$ observations on $p$ explanatory variables assumed to be of full column rank, $\beta$ is a $p \times 1$ vector of regression coefficients associated with them and $u$ is an $n \times 1$ vector of disturbances, the elements of which are assumed to be i.i.d. with

$$
E(u)=0 ; \operatorname{Var}(u)=\sigma^{2} I .
$$

The OLSE for $\beta$ in model (2) is given by

$$
\hat{\beta}_{O L S E}=\left(X^{\prime} X\right)^{-1} X^{\prime} y
$$

that is well known to be the best linear unbiased estimator. As stated earlier, OLSE may result in large sampling variances in the presence of multicollinearity and therefore may produce estimators which are not in tune with the researcher's prior belief. To deal with this problem, Hoerl and Kennard [1] proposed the ridge regression estimator by allowing some bias into the estimator which resulted in smaller MSE. The estimator is given by

$$
\hat{\beta}_{R R E}=\left(X^{\prime} X+k I\right)^{-1} X^{\prime} y=\left(I-k A^{-1}\right) \hat{\beta}_{O L S E},
$$

where $k \geq 0$ and $A=X^{\prime} X+k I$.

Later, in order to reduce the problem of bias associated with RRE, Singh et al. [7] proposed an almost unbiased ridge estimator using the Jackknife technique that was introduced by Quenouille [8] as a general method for reducing the bias of an estimator. Later Tukey [9] proposed that this technique may also offer a simple method to obtain the confidence intervals for the parameters of interest. The estimator given by Singh et al. [7] is called the jackknifed ridge estimator (JRE) and it is given by

$$
\hat{\beta}_{J R E}=\left[I-\left(k A^{-1}\right)^{2}\right] \hat{\beta}_{O L S E}
$$

The application of the jackknife technique in this context was also investigated in Nyquist [10], independently for bias reduction, variance estimation and tracing of influential observations. For recent developments in jackknifed ridge estimator, see Khurana et. al. [11]. 
Now for bootstrapping, firstly, for the model defined in (2), we fit the least squares regression equation for full sample, calculate the standardized residuals $\hat{u}_{i}$ and then draw an $n$ sized bootstrap sample with replacement $\left(\hat{u}_{1}^{(b)}, \hat{u}_{2}^{(b)}, \ldots, \hat{u}_{n}^{(b)}\right)$ from the residuals $\hat{u}_{i}$ 's giving $1 / n$ probability to each $\hat{u}_{i}$. After this, we obtain the bootstrap $y$ values using the resampled residuals keeping the design matrix fixed as shown below

$$
y^{(b)}=X \hat{\beta}_{O L S E}+\hat{u}^{(b)} .
$$

We then regress these bootstrapped $y$ values on the fixed $X$ to obtain the bootstrap estimates of the regression coefficients. So, the RRE from the first bootstrap sample is

$$
\hat{\beta}_{R R E(b 1)}^{*}=\left(X^{\prime} X+k I\right)^{-1} X^{\prime} y_{(b 1)} .
$$

Repeating the above steps $B$ times, where $B$ is the number of bootstrap samples, the bootstrap RRE for $\beta$ is given by

$$
\overline{\hat{\beta}}_{R R E}^{*}=\sum_{r=1}^{B} \hat{\beta}_{R R E(b r)}^{*} / B
$$

The estimated bias is given by

$$
\text { Bias }_{\text {est }}=\overline{\hat{\beta}}_{R R E}^{*}-\hat{\beta}_{O L S E}
$$

The estimated variance of RRE through bootstrap is given as

$$
\operatorname{Var}_{e s t}=\sum_{r=1}^{B}\left(\hat{\beta}_{R R E(b r)}^{*}-\overline{\hat{\beta}}_{R R E}^{*}\right)^{2} /(B-1)
$$

Now, based on these estimates, we construct the confidence intervals for the regression coefficient $\beta$ in following subsection.

\subsection{Confidence Intervals for Regression Coefficients using RRE}

There are several methods for constructing bootstrap confidence intervals based on the estimate of variance given in (7) are described briefly below.

\subsubsection{Normal theory method}

The first method for constructing bootstrap confidence interval is based on the assumption that the sampling distribution of $\hat{\beta}_{R R E}$ is normal. A $95 \%$ confidence interval for $\beta$ based on RRE is

$$
\left(2 \hat{\beta}_{R R E}-\overline{\hat{\beta}}_{R R E}^{*}\right)-z_{(1-\alpha / 2)} \sqrt{\operatorname{Var}_{e s t}}<\beta<\left(2 \hat{\beta}_{R R E}-\overline{\hat{\beta}}_{R R E}^{*}\right)+z_{(1-\alpha / 2)} \sqrt{\operatorname{Var}_{e s t}},
$$

where $\alpha=0.05$, Var $_{\text {est }}$ is the bootstrap estimate of the variance of $\hat{\beta}_{R R E}$ as defined in (7) and $z_{(1-\alpha / 2)}$ is the $(1-\alpha / 2)$ quantile of the standard normal distribution. 


\subsubsection{Percentile method}

Another method for the construction of bootstrap confidence intervals is the bootstrap percentile method which is the most popular among all primarily due to its simplicity and natural appeal. In this method, we use the empirical quantiles of $\hat{\beta}_{R R E}^{*}$ to form the confidence interval for $\beta$. A $95 \%$ confidence interval for 1000 bootstrap resamples is

$$
\hat{\beta}_{R R E(25)}^{*}<\beta<\hat{\beta}_{R R E(975)}^{*},
$$

where $\hat{\beta}_{R R E(r)}^{*}$ is the $r^{\text {th }}$ observation in the ordered bootstrap replicates of $\hat{\beta}_{R R E}^{*}$.

\subsubsection{Studentized- $t$ method}

Another method for constructing the bootstrap confidence intervals is the studentized bootstrap, also called the bootstrap- $t$ method. The studentized- $t$ bootstrap confidence interval takes the same form as the normal confidence interval except that instead of using the quantiles from a normal distribution, the quantiles are calculated using the bootstrapped $t$-distribution (see Davison and Hinkley [12] and Efron and Tibshirani [13]). Bootstrapping a statistical function of the form $t=\left(\hat{\beta}_{R R E}-\right.$ $\beta) /(S E)$, where $S E$ is the sample estimate of the standard error of $\hat{\beta}_{R R E}$ results in extra accuracy (see Hall [14]). The bootstrap form of this is given by

$$
t^{*}=\left(\hat{\beta}_{R R E}^{*}-\hat{\beta}_{R R E}\right) /(S E)^{*},
$$

where $S E^{*}$ is the standard error based on bootstrap distribution. Denote the $100 s^{\text {th }}$ bootstrap percentile of $t^{*}$ by $b_{s}$ and consider the statement that $\left(b_{0.025}<t<b_{0.975}\right)$ and after substituting $t=\left(\hat{\beta}_{R R E}-\beta\right) / S E$, we get confidence limits for $\beta$ as

$$
\hat{\beta}_{R R E}-(S E) b_{0.975}<\beta<\hat{\beta}_{R R E}-(S E) b_{0.025} .
$$

This interval is known as bootstrap- $t$ based confidence interval for $\beta$ at $95 \%$ confidence level. The use of studentized bootstrap is not suitable for some cases mostly where the endpoints of the intervals are too wide or the outliers are present.

On the other hand, percentile bootstrap endpoints are simple to calculate and can work well, especially if the sampling distribution is symmetrical but it may not have the correct coverage when the sampling distribution of the statistic is skewed. Its coverage can be improved by adjusting the endpoints for bias. This method is known as Bias corrected accelerated (BCa) method which we discuss in the next subsection.

\subsubsection{BCa method}

If we have a distribution which is skewed, few adjustments are required. One method which is proved to be reliable in such cases is BCa method, this method will tend to be closer to the true confidence interval than the percentile method. For a detailed review on the same, see Efron [4], Hall [5], Efron and Tibshirani [13]. 
The $\mathrm{BCa}$ procedure approximates confidence intervals for $\beta$ from the percentiles of the bootstrap histogram. $\hat{\beta}_{R R E}$ is an estimate of $\beta$ based on the observed data and $\hat{\beta}_{R R E}^{*}$ is a bootstrap replication of $\hat{\beta}_{R R E}$ obtained by resampling. Let $\hat{G}(c)$ be the cumulative distribution function of B bootstrap replications of $\hat{\beta}_{R R E}^{*}$,

$$
\hat{G}(c)=\#\left\{\hat{\beta}_{R R E}^{*}<c\right\} / B .
$$

The upper endpoint, $\hat{\beta}_{R R E(B C a)}[\alpha]$ of the one-sided confidence interval at $\alpha$ level i.e. $\beta \in\left(-\infty, \hat{\beta}_{R R E(B C a)}[\alpha]\right)$ is defined in terms of $\hat{G}$ and two parameters $z_{0}$, the bias correction and $a$, the acceleration. The BCa endpoint is given by

$$
\hat{\beta}_{R R E(B C a)}[\alpha]=\hat{G}^{-1} \Phi\left(z_{0}+\frac{z_{0}+z^{(\alpha)}}{1-a\left(z_{0}+z^{(\alpha)}\right)}\right) .
$$

Here $\Phi$ is the standard normal distribution function, with $z^{(\alpha)}=\Phi^{-1}(\alpha)$. The central $90 \%$ BCa confidence interval is given by $\left(\hat{\beta}_{R R E(B C a)}[0.05], \hat{\beta}_{R R E(B C a)}[.95]\right)$. In (9), if $a$ and $z_{0}$ are zero, then $\hat{\beta}_{R R E(B C a)}[\alpha]=\hat{G}^{-1}(\alpha)$, the $100 \alpha^{\text {th }}$ percentile of the bootstrap replications. Also, if $\hat{G}$ is normal, then $\hat{\beta}_{R R E(B C a)}[\alpha]=\hat{\beta}_{R R E}+z^{(\alpha)} \hat{\sigma}$, the standard interval endpoint. In general, (9) makes three different corrections to the standard intervals, improving their coverage accuracy.

The $\mathrm{BCa}$ algorithm estimates $z_{0}$ by

$$
\hat{z_{0}}=\Phi^{-1}\left\{\frac{\#\left\{\hat{\beta}_{R R E}^{*}(b)<\hat{\beta}_{R R E}\right\}}{B}\right\}
$$

The acceleration $a$ is estimated as

$$
\hat{a}=\frac{1}{6} \frac{\sum_{i=1}^{n} U_{i}^{3}}{\left(\sum_{i=1}^{n} U_{i}^{2}\right)^{3 / 2}}
$$

For calculating $U_{i}$, we can use the following jackknife influence function (see Hinkley [15]) in (10)

$$
U_{i}=(n-1)\left(\hat{\beta}_{R R E}-\hat{\beta}_{R R E(i)}\right),
$$

where $\hat{\beta}_{R R E(i)}$ is the estimate of $\beta$ based on the reduced data set by removing the $i^{t h}$ observation from the data.

Another method known as the ABC (approximate bootstrap confidence intervals) method was proposed by Efron[4], that gives analytic adjustment to $\mathrm{BCa}$ method for smoothly defined parameters in exponential families. They are touted in the literature as improvements for common parametric and non-parametric BCa procedures, and may be preferred in order to avoid the BCa's Monte Carlo calculations (see DiCiccio and Efron [16]; Efron and Tibshirani [13]; DiCiccio and Efron [17]). The authors adopted this method in the linear model setup, however did not notice any significant improvement in its performance over the $\mathrm{BCa}$ method. Hence, this method has not been not pursued in the numerical investigations carried out. 


\subsubsection{Jackknife method}

Jackknife technique is generally used to reduce the bias of parameter estimates and to estimate the variance. A $95 \%$ jackknife confidence interval for $\beta$ based on RRE is

$$
\hat{\beta}_{J R E}-t\left(1-\frac{\alpha}{2} ; n-p\right) \sqrt{v_{i i}}<\beta<\hat{\beta}_{J R E}+t\left(1-\frac{\alpha}{2} ; n-p\right) \sqrt{v_{i i}},
$$

where $\alpha=0.05, t\left(1-\frac{\alpha}{2} ; n-p\right)$ is the upper $\frac{\alpha}{2} \times 100 \%$ point of the Students $t$-distribution with $(n-p)$ degrees of freedom and $v_{i i}$ is the $i^{t h}$ diagonal element of the following variance estimate of RRE.

$$
\hat{\operatorname{Var}}_{J}\left(\hat{\beta}_{R R E}\right)=\frac{1}{n(n-p)} \sum_{i=1}^{n}\left(Q_{i}-\hat{\beta}_{J R E}\right)\left(Q_{i}-\hat{\beta}_{J R E}\right)^{\prime}
$$

where $Q_{i}{ }^{\prime} s$ are the pseudo values defined as

$$
Q_{i}=\hat{\beta}_{R R E}+n\left(1-w_{i}\right)\left(\hat{\beta}_{R R E}-\hat{\beta}_{R R E(-i)}\right)
$$

where $\hat{\beta}_{R R E(-i)}$ is RRE calculated by deleting the $i^{\text {th }}$ row from the data and $w_{i}=x_{i}{ }^{\prime} A^{-1} x_{i}$ where $A$ is as defined earlier and $x_{i}^{\prime}$ is the $i^{\text {th }}$ row of $X$ matrix.

In order to compare these methods of constructing asymptotic confidence intervals based on RRE and OLSE, coverage probabilities which is defined as the proportion that the confidence interval includes the true parameter, under repeated sampling from the same underlying population and confidence width which is the difference between the upper and lower confidence endpoints have been calculated. In the next section, a simulation study to obtain the confidence widths and coverage probabilities based on the confidence intervals developed using RRE and OLSE has been carried out.

\section{A simulation study}

After getting into some theoretical aspects of each method to construct confidence intervals, the methods are applied on simulated data and their performance is compared based on the coverage probabilities and confidence widths. The model is

$$
y=X \beta+u
$$

where $u \sim N(0,1)$. Here $\beta$ is taken as the normalized eigen vector corresponding to the largest eigen value of $X^{\prime} X$. The explanatory variables are generated from the following equation

$$
x_{i j}=\left(1-\rho^{2}\right)^{\frac{1}{2}} w_{i j}+\rho w_{i p}, i=1,2 \ldots, n ; j=1,2, \ldots, p .
$$

where $w_{i j}$ are independent standard normal pseudo-random numbers and $\rho^{2}$ is the correlation between the two explanatory variables for $j, j^{\prime}<p$ and $j \neq j^{\prime}$. When $j$ or $j^{\prime}=p$, the correlation will be $\rho$. Two different values of $\rho$ are taken as $\rho=0.9$ and 0.99 to investigate the effects of different degrees of collinearity with sample sizes $n=25,50$ and 100 . The feasible 
value of $k$ is obtained by the optimal formula $k=\frac{p \sigma^{2}}{\beta^{\prime} \beta}$ as given by Hoerl et al. [18], so that

$$
\hat{k}=\frac{p \hat{\sigma}^{2}}{\hat{\beta}_{O L S E}^{\prime} \hat{\beta}_{O L S E}}
$$

where

$$
\hat{\sigma}^{2}=\frac{\left(y-X \hat{\beta}_{\text {OLSE }}\right)^{\prime}\left(y-X \hat{\beta}_{\text {OLSE }}\right)}{n-p} .
$$

For calculating different bootstrap confidence intervals like Normal, Percentile, Studentized and BCa, the function called 'boot.ci' in R is used. The confidence limits through jackknife are calculated using (11). The coverage probabilities and average confidence width using 1999 bootstrap resamples are calculated and the experiment is repeated 1000 times. The coverage probability, say $\mathrm{CP}$ is calculated using the following formula

$$
C P=\frac{\#\left(\hat{\beta}_{L}<\beta<\hat{\beta}_{U}\right)}{N},
$$

and the average confidence width, say $\mathrm{CW}$ is calculated by

$$
C W=\frac{\sum_{i=1}^{N}\left(\hat{\beta}_{U}-\hat{\beta}_{L}\right)}{N}
$$

where $N$ is the simulation size, $\hat{\beta}_{L}$ and $\hat{\beta}_{U}$ are lower and upper confidence interval endpoints respectively. The results for coverage probability and average confidence width at $95 \%$ and $99 \%$ confidence levels with different values of $n$ and $\rho$ are summarized in Table 1-Table 4. Note that in all the tables, column namely OLSE gives the coverage probability and confidence width based on the confidence intervals through $\hat{\beta}_{O L S E}$ using $t$-distribution.

From Tables 1 and 2, it is found that the coverage probabilities of all the intervals improve with the increasing value of $n$ and become close to each other which is due to the consistency of the estimators. It is evident from Tables 3 and 4 that the confidence intervals based on RRE have shorter widths in comparison to the width of the interval based on OLSE.

It is interesting to note that the coverage probabilities and confidence widths through OLSE and through jackknife are very close to each other. Also, from Tables 3 and 4, it can be seen that with the increasing collinearity between the dependent variables, the difference between the confidence width of interval based on OLSE and intervals based on RRE is increasing. Also, with the increasing value of sample size, the confidence width of all the intervals is decreasing.

According to Tables 1 and 2, all the bootstrap methods are generally conservative in terms coverage probabilities, however jackknife method seems to give coverage probabilities closer to the target. In terms of confidence width, resampling methods have smaller confidence width than the OLSE; jackknife method having larger confidence width than bootstrap methods. In noting that bootstrap methods are conservative with smaller confidence width, they seem to have an advantage over the jackknife method.

\section{Conclusion}

In the present study, the use of different confidence intervals based on bootstrap and jackknife resampling methods is illustrated. We computed the coverage probabilities and confidence width based on RRE using different bootstrap and 
jackknife methods based on a simulation study and compared it with that of OLSE which we computed using $t$-intervals. The shorter confidence widths obtained through RRE show its superiority over OLSE in the case of multicollinearity. Bootstrap methods produces intervals having shorter width than that of intervals produced by jackknife. This shows that the bootstrap method has a slight edge over the jackknife method.

Table 1: Coverage Probabilities through different methods at $95 \%$ confidence level

\begin{tabular}{c|c|c|c|c|c|c|c}
\hline$n$ & $\rho$ & OLSE & Normal & Percentile & Studentized & BCa & Jackknife \\
\hline 25 & 0.9 & 0.952 & 0.966 & 0.974 & 0.978 & 0.970 & 0.940 \\
& & 0.964 & 0.966 & 0.960 & 0.976 & 0.964 & 0.922 \\
& & 0.962 & 0.972 & 0.978 & 0.986 & 0.976 & 0.944 \\
\cline { 2 - 8 } & 0.99 & 0.952 & 0.970 & 0.980 & 0.986 & 0.972 & 0.940 \\
& & 0.964 & 0.964 & 0.964 & 0.984 & 0.966 & 0.920 \\
& & 0.964 & 0.986 & 0.986 & 0.994 & 0.990 & 0.936 \\
\hline 50 & 0.9 & 0.950 & 0.974 & 0.972 & 0.980 & 0.970 & 0.948 \\
& & 0.952 & 0.968 & 0.976 & 0.984 & 0.976 & 0.936 \\
& & 0.948 & 0.988 & 0.986 & 0.990 & 0.990 & 0.964 \\
\cline { 2 - 8 } & 0.99 & 0.950 & 0.984 & 0.984 & 0.986 & 0.984 & 0.948 \\
& & 0.952 & 0.986 & 0.984 & 0.990 & 0.986 & 0.934 \\
& & 0.948 & 0.994 & 0.994 & 0.996 & 0.994 & 0.968 \\
\hline 100 & 0.9 & 0.958 & 0.972 & 0.974 & 0.978 & 0.974 & 0.948 \\
& & 0.962 & 0.958 & 0.954 & 0.956 & 0.952 & 0.930 \\
& & 0.940 & 0.966 & 0.968 & 0.968 & 0.964 & 0.932 \\
\cline { 2 - 8 } & 0.99 & 0.958 & 0.984 & 0.984 & 0.984 & 0.984 & 0.946 \\
& & 0.962 & 0.974 & 0.976 & 0.982 & 0.974 & 0.928 \\
& & 0.944 & 0.978 & 0.978 & 0.980 & 0.978 & 0.926 \\
\hline
\end{tabular}

Table 2: Coverage Probabilities through different methods at $99 \%$ confidence level

\begin{tabular}{c|c|c|c|c|c|c|c}
\hline$n$ & $\rho$ & OLSE & Normal & Percentile & Studentized & BCa & Jackknife \\
\hline 25 & 0.9 & 0.992 & 0.992 & 0.990 & 0.994 & 0.990 & 0.984 \\
& & 0.990 & 0.990 & 0.988 & 0.994 & 0.992 & 0.982 \\
& & 0.996 & 1.000 & 0.998 & 1.000 & 0.996 & 0.994 \\
\cline { 2 - 8 } & 0.99 & 0.992 & 0.992 & 0.990 & 0.994 & 0.992 & 0.984 \\
& & 0.990 & 0.990 & 0.992 & 0.996 & 0.992 & 0.978 \\
& & 0.998 & 0.996 & 1.000 & 1.000 & 0.998 & 0.992 \\
\hline 50 & 0.9 & 0.998 & 0.994 & 0.994 & 0.994 & 0.994 & 0.988 \\
& & 0.990 & 0.994 & 0.996 & 0.998 & 0.996 & 0.990 \\
& & 0.992 & 0.994 & 0.996 & 0.996 & 0.994 & 0.994 \\
\cline { 2 - 8 } & 0.99 & 0.998 & 0.994 & 0.994 & 0.994 & 0.994 & 0.988 \\
& & 0.990 & 1.000 & 0.998 & 1.000 & 0.998 & 0.990 \\
& & 0.988 & 0.998 & 1.000 & 1.000 & 0.998 & 0.994 \\
\hline 100 & 0.9 & 0.988 & 0.992 & 0.992 & 0.992 & 0.992 & 0.988 \\
& & 0.990 & 0.988 & 0.986 & 0.990 & 0.988 & 0.982 \\
& & 0.994 & 0.990 & 0.988 & 0.994 & 0.986 & 0.976 \\
\cline { 2 - 8 } & 0.99 & 0.988 & 0.992 & 0.992 & 0.994 & 0.992 & 0.988 \\
& & 0.990 & 0.996 & 0.994 & 0.998 & 0.996 & 0.980 \\
& & 0.992 & 0.996 & 0.996 & 0.996 & 0.992 & 0.978 \\
\hline
\end{tabular}


Table 3: Average confidence width through different methods at 95\% confidence level using OLSE and RRE

\begin{tabular}{c|c|c|c|c|c|c|c}
\hline$n$ & $\rho$ & OLSE & Normal & Percentile & Studentized & BCa & Jackknife \\
\hline 25 & 0.9 & 2.101518 & 1.1330 & 1.1346 & 1.2801 & 1.1361 & 1.6494 \\
& & 1.998510 & 1.1712 & 1.1732 & 1.3216 & 1.1738 & 1.6516 \\
& & 2.144994 & 1.0454 & 1.0475 & 1.1809 & 1.0483 & 1.5851 \\
\cline { 2 - 8 } & 0.99 & 6.493567 & 2.3355 & 2.3407 & 2.6376 & 2.3437 & 3.6672 \\
& & 6.175280 & 2.3954 & 2.4000 & 2.7049 & 2.4008 & 3.6976 \\
& & 7.994216 & 2.2116 & 2.2146 & 2.5011 & 2.2178 & 3.7170 \\
\hline 50 & 0.9 & 1.407747 & 0.9846 & 0.9864 & 1.0449 & 0.9868 & 1.2858 \\
& & 1.258123 & 0.9225 & 0.9241 & 0.9793 & 0.9245 & 1.1733 \\
& & 1.188554 & 0.8173 & 0.8185 & 0.8671 & 0.8191 & 1.0739 \\
\cline { 2 - 8 } & 0.99 & 4.349856 & 1.7202 & 1.7245 & 1.8256 & 1.7251 & 2.6731 \\
& & 3.887527 & 1.7090 & 1.7124 & 1.8153 & 1.7128 & 2.5918 \\
& & 4.654095 & 1.5836 & 1.5854 & 1.6813 & 1.5869 & 2.5555 \\
\hline 100 & 0.9 & 0.9346568 & 0.7506 & 0.7518 & 0.7732 & 0.7518 & 0.8923 \\
& & 0.8637652 & 0.7042 & 0.7053 & 0.7244 & 0.7057 & 0.8282 \\
& & 0.9818960 & 0.7410 & 0.7423 & 0.7639 & 0.7430 & 0.9207 \\
\cline { 2 - 8 } & 0.99 & 2.888035 & 1.3929 & 1.3957 & 1.4355 & 1.3971 & 1.9978 \\
& & 2.668984 & 1.3652 & 1.3669 & 1.4057 & 1.3687 & 1.9255 \\
& & 3.754617 & 1.3487 & 1.3496 & 1.3918 & 1.3509 & 2.1479 \\
\hline
\end{tabular}

Table 4: Average confidence width through different methods at 99\% confidence level using OLSE and RRE

\begin{tabular}{c|c|c|c|c|c|c|c}
\hline$n$ & $\rho$ & OLSE & Normal & Percentile & Studentized & BCa & Jackknife \\
\hline 25 & 0.9 & 2.856330 & 1.4890 & 1.4908 & 1.7480 & 1.4960 & 2.2419 \\
& & 2.716325 & 1.5392 & 1.5341 & 1.8025 & 1.5378 & 2.2449 \\
& & 2.915421 & 1.3739 & 1.3774 & 1.6143 & 1.3819 & 2.1544 \\
\cline { 2 - 8 } & 0.99 & 8.825893 & 3.0694 & 3.0764 & 3.6005 & 3.0870 & 4.9844 \\
& & 8.393285 & 3.1480 & 3.1353 & 3.6944 & 3.1443 & 5.0257 \\
& & 10.865537 & 2.9065 & 2.9123 & 3.4147 & 2.9281 & 5.0521 \\
\hline 50 & 0.9 & 1.878560 & 1.2940 & 1.3010 & 1.4038 & 1.3030 & 1.7159 \\
& & 1.678895 & 1.2124 & 1.2190 & 1.3119 & 1.2195 & 1.5658 \\
& & 1.586059 & 1.0741 & 1.0794 & 1.1616 & 1.0804 & 1.4331 \\
\cline { 2 - 8 } & 0.99 & 5.804641 & 2.2607 & 2.2739 & 2.4573 & 2.2757 & 3.5671 \\
& & 5.187688 & 2.2460 & 2.2573 & 2.4294 & 2.2602 & 3.4586 \\
& & 6.210630 & 2.0813 & 2.0925 & 2.2534 & 2.0943 & 3.4102 \\
\hline \multirow{2}{*}{100} & 0.9 & 1.237342 & 0.987 & 0.991 & 1.029 & 0.991 & 1.181 \\
& & 1.143492 & 0.925 & 0.931 & 0.962 & 0.933 & 1.096 \\
& & 1.299879 & 0.974 & 0.979 & 1.017 & 0.979 & 1.219 \\
\cline { 2 - 8 } & 0.99 & 3.823313 & 1.831 & 1.839 & 1.908 & 1.841 & 2.645 \\
& & 3.533324 & 1.794 & 1.803 & 1.867 & 1.810 & 2.549 \\
& & 4.970534 & 1.772 & 1.782 & 1.853 & 1.782 & 2.844 \\
\hline
\end{tabular}

\section{Competing interests}

The authors declare that they have no competing interests. 


\section{Authors' contributions}

All authors have contributed to all parts of the article. All authors read and approved the final manuscript.

\section{References}

[1] Hoerl AE, Kennard RW. Ridge regression: Biased estimation for nonorthogonal problems. Technometrics. 1970, $12(1): 55-67$.

[2] Firinguetti L, Bobadilla G. Asymptotic confidence intervals in ridge regression based on the edgeworth expansion. Statistical Papers. 2011, 52(2):287-307.

[3] Crivelli A, Firinguetti L, Montano R, Munóz M. Confidence intervals in ridge regression by bootstrapping the dependent variable: a simulation study. Communications in Statistics-Simulation and Computation. 1995, 24(3):631-52.

[4] Efron B. Better bootstrap confidence intervals. Journal of the American statistical Association. 1987, 82(397):171-85.

[5] Hall P. Theoretical comparison of bootstrap confidence intervals. The Annals of Statistics. 1988, 16(3):927-53.

[6] DiCiccio T, Tibshirani R. Bootstrap confidence intervals and bootstrap approximations. Journal of the American Statistical Association. 1987, 82(397):163-70.

[7] Singh B, Chaubey YP, Dwivedi TD. An almost unbiased ridge estimator. Sankhy?: The Indian Journal of Statistics, Series B. 1986, 48(3):342-346.

[8] Quenouille MH. Notes on bias in estimation. Biometrika. 1956, 43(3/4):353-60.

[9] Tukey JW. Bias and confidence in not-quite large samples. Ann. Math. Statist.. 1958;29:614.

[10] Nyquist H. Applications of the jackknife procedure in ridge regression. Computational Statistics \& Data Analysis. 1988, 6: 177183.

[11] Khurana M, Chaubey YP, Chandra S. Jackknifing the ridge regression estimator: A revisit. Communications in Statistics-Theory and Methods. 2014, 43(24):5249-62.

[12] Davison AC, Hinkley DV. Bootstrap methods and their application. Cambridge university press; 1997.

[13] Efron B, Tibshirani RJ. An introduction to the bootstrap. CRC press; 1994.

[14] Hall P. The bootstrap and Edgeworth expansion. Springer Science \& Business Media; 2013.

[15] Hinkley DV. Jackknifing in unbalanced situations. Technometrics. 1977, 19(3):285-92.

[16] DiCiccio TH, Efron B. More accurate confidence intervals in exponential families. Biometrika. 1992, 79(2):231-45.

[17] DiCiccio TJ, Efron B. Bootstrap confidence intervals. Statistical Science. 1996, 11(3):189-212.

[18] Hoerl AE, Kannard RW, Baldwin KF. Ridge regression: some simulations. Communications in Statistics-Theory and Methods. 1975, 4(2):105-23. 